\title{
424. Primäre Knochenheilung an der Rippe
}

\author{
K. E. Rehm, K. H. Schultheis und K. Kunze \\ Zentrum für Chirurgie der Justus-Liebig-Universität, D-6300 Gießen

\section{Primary Healing of Rib Fractures}

Summary. The principles of internal stabilization are also valid in chest wall stabilization. Because physiological load cannot be interrupted, high stability is necessary. After measurement of the dynamic force at the rib fracture in vivo and experimental fractures of cadaver ribs, a new implant was developed. With this, primary healing is possible both in the osseous and in the cartilaginous parts of the rib and sternum. This was demonstrated in animal experiments and clinical cases. The biomechanical data of ribs and some typical methods of stabilization are listed. The recommended implants restore the physiological rates of tensile strength and elasticity. Roentgenographs and histological findings demonstrate that primary healing of rib fractures is possible.

Key words: Flail chest - Internal stabilization - Primary healing.

Zusammenfassung. Für die Thoraxwandstabilisierung gelten die Prinzipien der Osteosynthese wie am übrigen Skelet. Da die physiologische Belastung nicht unterlassen werden kann, muß Belastungsstabilität erreicht werden. Nach Messung der dynamischen Kräfte an der durchtrennten Rippe des Lebenden und Bruchversuchen an Leichenrippen wurden Implantate entwickelt, welche sowohl im knorpeligen als auch im knöchernen Bereich der Rippe ebenso wie am Sternum eine primäre Spaltheilung ermöglichen. Dies wurde tierexperimentell und klinisch nachgewiesen. Die biomechanischen Daten von Rippen und einige typische Osteosynthesen werden tabellarisch dargestellt. Die neu vorgestellten Implantate stellen die physiologischen Ausgangswerte von Federkonstante und Bruchlast wieder her. Damit konnte histologisch und röntgenologisch der Beweis für die primäre Knochenheilung an der Rippe erbracht werden.

Schliusselwörter: Instabiler Thorax - Thoraxwandstabilisierung - Primäre Knochenheilung.

\section{Die Anlagerung von autologen Rippenspänen zur Überbrückung langstreckiger Knochendefekte}

\author{
H. Ecke, W. Völkel, L. Faupel und A. Schulz \\ Unfallchirurgische Universitätsklinik der Justus-Liebig-Universität Gießen (Leiter: Prof. Dr. H. Ecke), \\ D-6300 Gießen
}

\section{Apposition of Autogenous Split-Rib Grafts for Repair of Extensive Bone Defects}

Summary. The use of autogenous cortico-cancellous bone grafts to repair bone defects, to treat nonunion of fractures, and after continuity resection has proved to be a valuable technique. An additional method that can be used to fill extensive defects of bones is the apposition of corticocancellous rib grafts. Pictures illustrate resected rib grafts, histological and radiological checks, and later blood flow in the graft. The results of 42 appositions of autologous rib grafts are displayed in tables.

Key words: Autogenous split - Rib grafts.

Zusammenfassung. Der Wert der autologen Knochenspanverpflanzung bei Osteosynthesen von Frakturen, Pseudarthrosen und nach Kontinuitätsresektionen ist seit langem bekannt. Eine zusätzliche Bereicherung stellt der corticospongiöse Rippenspan dar. In einer Bildfolge werden die Technik der Spanentnahme, der Spaneinbau histologisch und röntgenologisch, sowie dessen spätere Durchblutung gezeigt. Die Ergebnisse von 42 autologen Rippenspananlagerungen werden tabellarisch dargestellt.

Schlïsselwörter: Autologe Rippenspäne. 